\title{
RETENTION AND WEAR CHARACTERISTIC ASSESSMENT OF OT EQUATOR WITH SMART BOX ATTACHMENT VERSUS BALL ATTACHMENT FOR MANDIBULAR TWO IMPLANT ASSISTED OVER DENTURE. IN-VITRO STUDY
}

\author{
Doaa Amr Rostom* and Nourhan Ahmed Ragheb ${ }^{* *}$
}

\begin{abstract}
Introduction: Excessive implant angulation, leads to increased maintenance of the prosthesis .This research aimed to evaluate the retentive power and wear of the equator with smart box attachment use with 10 degree tilted two implant assisted mandibular overdenture.
\end{abstract}

Material and methods:: this in-vitro was carried on six mandibular completely edentulous casts which received two mandibular implant in the cuspid region bilaterally .the cast divided into 2 equal group. (Groups I) OT equator with smartbox attachment and (Groups II) ball attachment. The overdenture were subjected to 1440 cyclic loads. The retention values and wear of the attachments were recorded baseline and after 0,720 , and 1440 cycles .

Result: Comparison between both groups was performed using Independent t-test which revealed significant difference after 1400 cycle $\mathrm{P}<0.05$ as equator with smart box group was significantly higher than ball group.

Conclusion: OT equator with smart box attachment should be preferred as the choice of attachment system for nonparallel implant overdenture.

KEYWORDS: Nonparallel implant, OT equator attachment, overdenture

\section{INTRODUCTION}

The key factors for successful implant retained overdentures treatment includes the number, location, and distribution of implants and choice of abutment. For optimal treatment, implant should be parallel to each other and perpendicular to the occlusal plane Some patients often present with challenging anatomical features range from straight or rounded to irregular and sharp ridges, and variability in bone width, insufficient bone volume in all dimensions, the inferior alveolar nerve being anteriorly or superiorly positioned and/or critical anatomy that make ideal placement of dental implants impossible. ${ }^{1-3}$

\footnotetext{
* Lecturer in Prosthodontics Department, Faculty of Dentistry, Cairo Univ.

** Lecturer in Prosthodontics Department, Faculty of Oral and Dental Medicine, Kafrelshiekh Univ.
} 
Placing the implant parallel to each other in such compromised cases required surgical correction but some patient's desire to avoid additional surgical procedures thus may prompt a clinician to angulate a dental implant, which could result in the positioning of implants in non-ideal configurations ${ }^{4}$.

Excessive implant angulation, increased maintenance and wear of inserts, and difficulty maintaining hygiene. In addition increased angulation may result in greater challenges to inserting and removing the prosthesis, which is unfavorable for patients who are older or have limited dexterity. ${ }^{5}$

Resilient Ball attachment most commonly used dental attachment because it is the simplest form of all stud attachments which widely used as its practical, effective, relatively low cost, less technique sensitive, ease of handling, minimal chair side time requirements and their possible applications with both root and implant-supported prostheses so it is used with non-splinted implants ${ }^{6}$

Clinical studies reported that, ball attachment loss the retention of the prostheses regularly on short intervals. decrease in retention occurred as a result of attachment abrasion and micro movements during the mastication process, especially if the implants are non-parallel A lack of parallelism of the implants creates considerable wear of the rubber rings in a relatively short time span. ${ }^{6,7}$

Rhein83USA's custom attachment abutment design service is ideal for matching difficult implant rehabilitation cases, developed Smart Box, which could be used with the OT Equator in patients of extreme divergences among the implants. The Smart Box has an inner tilting mechanism that enables a passive insertion with divergent implants up to 50 degrees $^{8}$

This research aimed to evaluate the retentive power and wear of the smart box attachment use with 10 degree tilted two implant assisted mandibular overdenture
According to the knowledge of the authors, there were no studies to date that compared ball and smart box equator attachment for mandibular two implant assisted over denture regarding retention and wear.

\section{MATERIAL AND METHODS}

The in vitro comparative study was registered in clinical trial Gov. the study was conducted at faculty of dentistry Cairo University. Sample size calculation was depending on a previous study as references ${ }^{19,20}$. According to these studies, the response within each subject group was normally distributed with standard deviation 5.1. If the true difference in the experimental and control means is 20.4, minimally the study needed 3 implant assisted overdenture in each group to be able to reject the null hypothesis that the population means of the experimental and control groups are equal with probability (power) 0.8 . The Type I error probability associated with this test of this null hypothesis is 0.05 . Every overdenture subjected to 1400 insertion and removal cycles where retention forces and wear were measured. Two different attachment system were investigated. The study designed into two parallel group, control group was 2 ball attachment (Dentium) retaining overdenture, the test group was 2 smart box equator(Rhien OT equator with smart box) retaining overdenture

The overdenture were subjected to 1440 vertical insertion-separation cycles. The retention values of the attachments were recorded baseline and after 0,720 , and 1440 cycles using the universal testing machine. In addition, digital microscope integrated with image analysis software used for analysis the attachments wear

Regarding Cast fabrication; Standard Epoxy resin casts for edentulous mandibular arch were picked up in the study. To replicate the resiliency of soft mucosal tissue of the edentulous mandible, the surface of the cast replica was covered by uniform layer of A- silicone based soft lining material gingival mimic (Mollosil, detax co. Ettlingen/germany). The 
surface of the epoxy resin mandibular replica was roughened with carbide bur to increase adhesion of gingival mask material, the cast replica was lightly painted with A-silicon adhesive and let to dry. Uniform layer of gingival mask material mix was applied on the surface of the cast, the thickness of the simulated mucosa was $2.5-3 \mathrm{~mm}$.

Two implant (Dentium) installed at the canine region $(4.2 \mathrm{~mm}$ width and $10 \mathrm{~mm}$ length) with Interimplant divergence 10 degrees made with a surveying milling machine. Tripoding was first made to ensure reproducible in the positioning of the test models on the surveyor.

Regarding overdenture fabrication. Epoxy resin casts were duplicated for stone casts fabrication of a total number of six over dentures, for each selected interimplant divergence angle were 10 degree, after denture processing and denture finishing, access openings were made on the lingual flange of mandibular denture near canine region to allow visualization of the attachments under the denture base and excess material to flow to pick-up the attachment .The two implants were connected to the attachments using the abutment hex driver and were torqued to $30 \mathrm{~N} / \mathrm{cm}$ with a torque wrench. The attachment metal housing of ball and equator were pickup in the overdenture with self-cure acrylic resin. Fig 1(A, B)

\section{Retention and wear assessment}

The overdenture subjected to several insertion and removal test .To perform the insertion-removal test, a programmable logic controlled equipment; the newly developed four stations multimodal ROBOTA chewing simulator integrated with thermo-cyclic protocol operated on servo-motor (Model Ach-09075dc-T, Ad-Tech Technology Co., Ltd., Germany).

Each overdenture gripped by Jakobe's chuck of the upper part of machine through inverted t-shaped auto-polymerizing acrylic resin (Caulk, Dentsply) centrally positioned horizontal bar between first molars to facilitate the aligning with the loading axis of machine and proper load distribution

The machine allowed the placement of the overdenture to its predetermined terminal position and its subsequent removal from the attachments, thus simulating the placement and removal of overdenture. The models with the over-denture were mounted in Teflon housing in the lower sample holder of the chewing simulator

The test conditions were maintained at room temperature $\left(23 \pm 2^{\circ} \mathrm{C}\right)$ and wet condition (distilled water). To analyze the data obtained during the simulation test, intervals every 720 and 1440 cycles were established, representing the simulated insertion and removal of the denture over 6months and 12 months, estimating that the patient would perform four removals and insertions of the PRDP per day. The chewing simulator performer $15 \mathrm{~mm}$ Vertical movement with $90 \mathrm{~mm} / \mathrm{s}$ rising speed. Fig 1C

The degree of the attachment retention evaluated by performed using Bluehill ${ }^{\circledR}$ Lite from Instron Instrument, where Each cast with its overdenture was fixed to the lower fixed compartment of a materials testing machine (Model 3345; Instron Instruments Ltd., USA) with a loadcell of $5 \mathrm{kN}$ and data were recorded using computer software (Bluehill Lite; Instron Instruments) .The sample was attached through centrally positioned horizontal inverted t-shaped auto-polymerizing acrylic resin bar between first molars to facilitate the aligning with the loading axis of machine and proper load distribution. A tensile load with pull out mode of force by gripping the vertical arm of resin bar by Jacob's chuck of upper compartment of materials testing machine at a crosshead speed of $5 \mathrm{~mm} / \mathrm{min}$. The load required to totally dislodge sample was recorded in Newton

The acrylic resin occlusal rims was attached on the universal testing machine. This ensured parallelism of the acrylic resin plate and the epoxy model. Therefore, a tensile power was applied perpendicularly to the occlusal plane as much as 
possible to simulate a denture's axially directed dislodging forces

After cyclic loading, Wear patterns were examined under digital microscope integrated with image analysis software and compared to a new similar extra to observe changes on the extra surfaces after tests with different retentive caps. Fig (2)

Data were presented as mean, standard deviation retention loss $\%$ in 1 tables \& 1 line chart. Statistical analysis was performed with SPSS 16 ( Package for Scientific Studies), Graph pad prism $\&$ windows excel. Exploration of the given data was performed using Shapiro-Wilk test and Kolmogorov-Smirnov test for normality which revealed that the significant level (P-value) was insignificant as $\mathrm{P}$-value $>0.05$ which indicated that alternative hypothesis was rejected, and the concluded data originated from normal distribution (parametric data) resembling normal Bell curve.

\section{RESULT}

In group I (equator with smart box), the mean retention \pm standard deviation decreased from $14.59 \pm 1.931$ at baseline to $10.91 \pm 1.915$ after 740 cycle

Then decreased again to $10.61 \pm 1.435$ after 1400 cycles. Comparison between different follow up retention was performed using One Way ANOVA which revealed insignificant difference between them as $\mathrm{P}>0.05$, followed by Tukey`s Post Hok test for multiple comparisons which revealed insignificant difference also as presented in table (1) and figure (3).

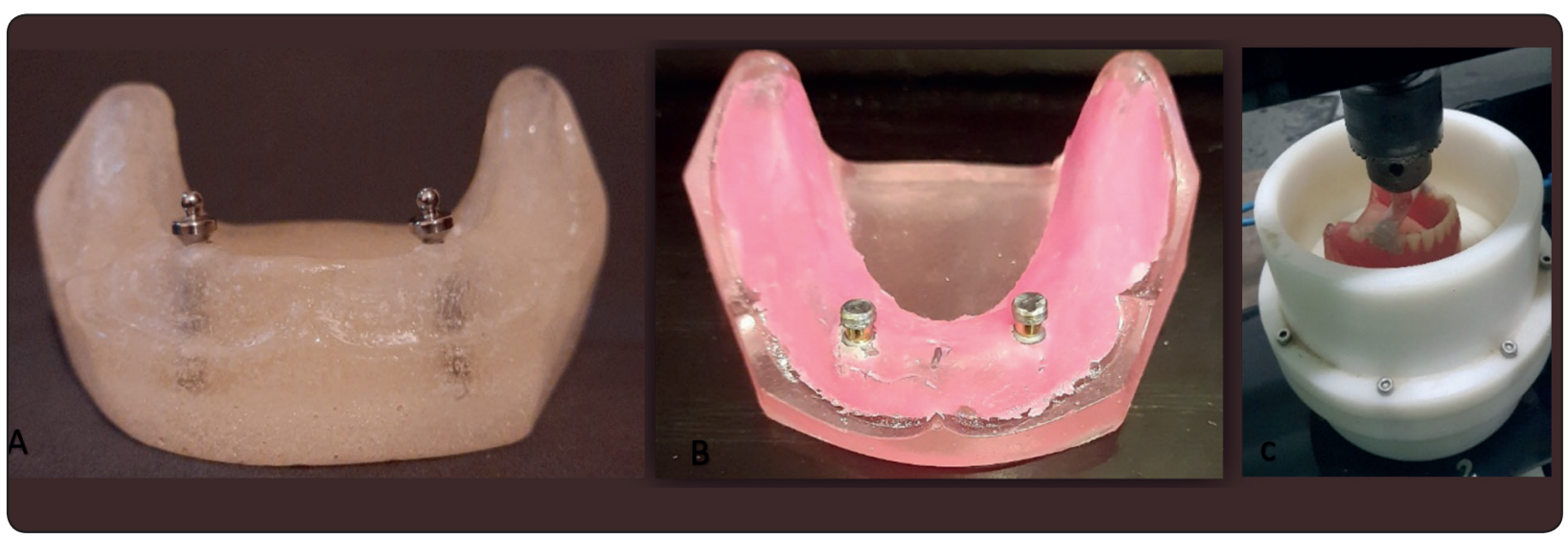

Fig (1): A: ball attachment. B: equator attachment with smart box. C: chewing simulator

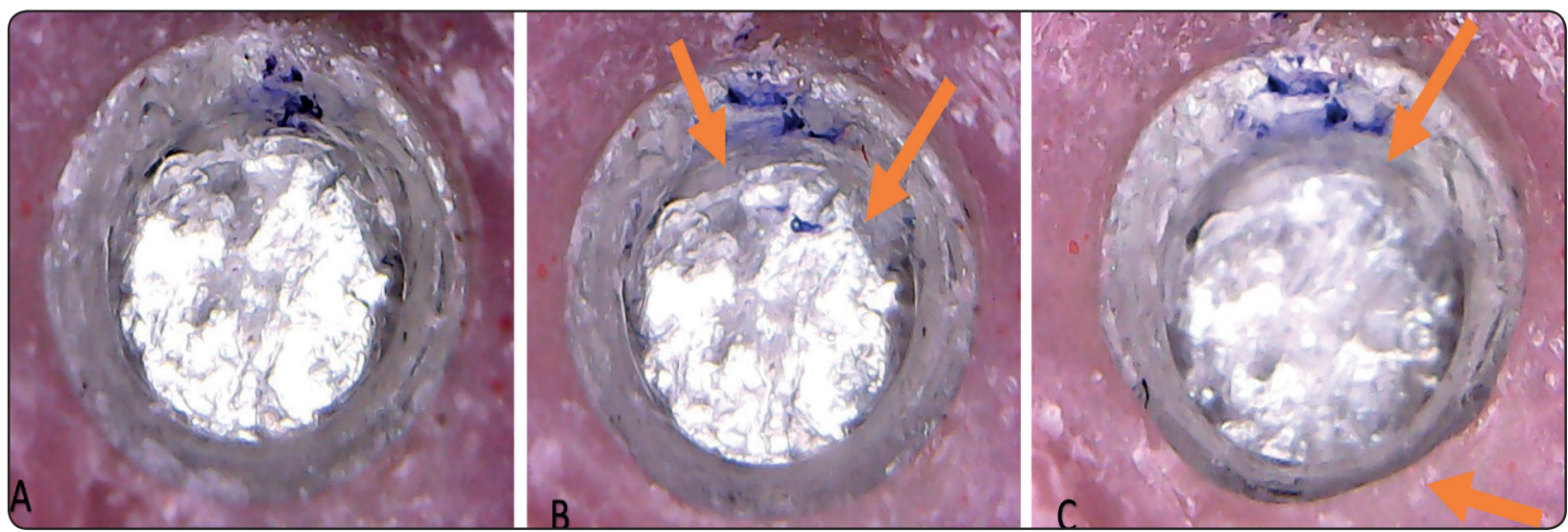

Fig (2): Surface deformation for the nylon cape of the female smart box nylon cap (A) baseline,(B) 740 cycles and (c) 1400 cycles 
TABLE (1) Mean, standard deviation and retention loss percentage in both groups at baseline, after 740,1400 cycles and comparison between them

\begin{tabular}{|c|c|c|c|c|c|}
\hline \multirow{2}{*}{ Time } & \multicolumn{2}{|c|}{ Equator smart box } & \multicolumn{2}{|c|}{ Ball } & \multirow{2}{*}{$P$ value } \\
\hline & M & $\pm \mathrm{SD}$ & M & $\pm \mathrm{SD}$ & \\
\hline Baseline & $14.59^{\mathrm{a}}$ & \pm 1.931 & $13.99^{\mathrm{a}}$ & \pm 1.859 & $0.71 \mathrm{~ns}$ \\
\hline 740 cycles & $10.91^{\text {a }}$ & \pm 1.915 & $7.11^{\mathrm{b}}$ & \pm 1.754 & $0.06 \mathrm{~ns}$ \\
\hline 1400cycles & $10.61^{\mathrm{a}}$ & \pm 1.435 & $5.21^{\mathrm{b}}$ & \pm 0.807 & $0.004 *$ \\
\hline $\mathrm{P}$ value & \multicolumn{2}{|c|}{$0.06 \mathrm{~ns}$} & \multicolumn{2}{|c|}{$0.0009 *$} & \\
\hline Retention loss \% & \multicolumn{2}{|c|}{-26.8} & \multicolumn{2}{|c|}{-62.5} & $0.42 \mathrm{~ns}$ \\
\hline
\end{tabular}

M; mean standard deviation

$\%$ percentage

Ns; insignificant difference

P; probability level.

* significant difference.

Means with the same superscript letters were insignificantly different P>0.05.

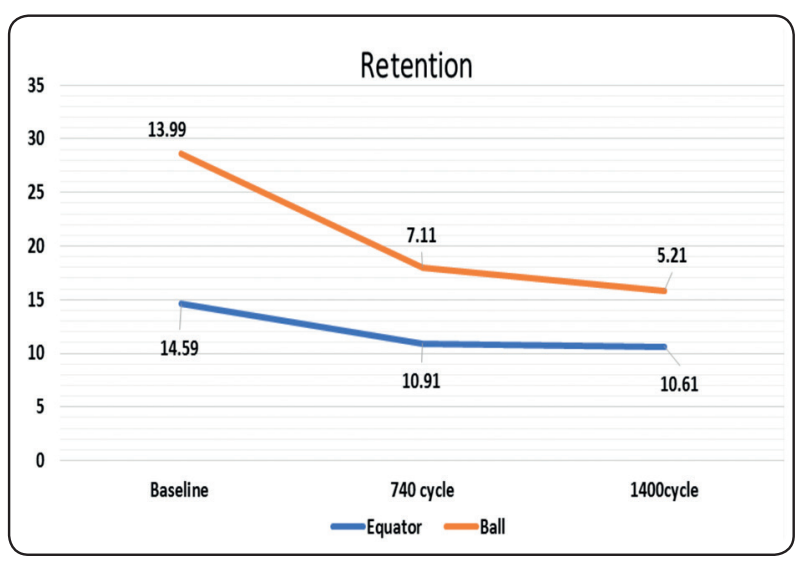

Fig. (3): Line chart represents retention of both group during follow up

In group II (Ball), the mean retention \pm standard deviation decreased from $13.99 \pm 1.859$ at baseline to $7.11 \pm 1.754$ after 740 cycle then decreased again to $15.21 \pm 0.807$ after 1400 cycle. Comparison between different follow up retention was performed using One Way ANOVA which revealed significant difference between them as $\mathrm{P}<0.05$, followed by Tukey`s Post Hok test for multiple comparisons which revealed significant difference between retention at baseline and retention after-740 cycle retention at baseline and retention after 1400 cycle, as presented in table (1) and figure (3).
Comparison between both groups was performed using Independent $t$-test which revealed insignificant difference $\mathrm{P}>0.05$ at baseline and after 740 cycle, while revealed significant difference after 1400 cycle $\mathrm{P}<0.05$ as equator group was significantly higher than ball group as presented in table (1) and figure (3).

Retention loss percentage was calculated according to the following formula: retention afterretention before / retention before $\times 100$. The retention loss of group I was lower than group II with insignificant difference between them by using Chi square test as presented in table (1).

Regarding wear characteristic. Dimensional changes of nylon parts were analyzed at the end of insertion and removal cycles after 1400 for both attachments system, concerning equator with smart box, the digital microscope shown minimal changes in inner diameter of the equator plastic part after 740 cyclic loading, and after 1400 cycles it showed inner and outer distortion

Wear simulation induced more surface rupture and distortion in ball attachment, there were notable change in the cap diameter in ball more than equator .The plastic cores underwent more dimensional changes after 1400 cycles equivalent to I year in ball attachment 


\section{DISCUSSION}

An overdenture placed in the mouth is subjected to a variety of forces applied in different directions. Retention is considered to be the force that resists withdrawal along the path of insertion and stabilizes the overdenture during function. ${ }^{8}$

The present in vitro study comparing the retention power and wear characteristic of the conventional most commonly used attachment ,ball attachment versus equator with smart box attachment because unfortunately there are no scientific articles comparing the performance of these attachment .

Many article proved that attachment retention loss is one of the most common post-insertion problem of implant overdenture, and this complication become obvious in case of nonparallel implants, as non-parallism interferes with the prosthesis path of insertion and removal and subsequently causes attachment wear. ${ }^{10,14}$

The final position of an implant can be altered because of the clinician's skill, patient cooperation, bone morphology, or guide stability. Some clinicians favor an angled abutment or a bar to compensate for implant angulation when the stud attachment is inappropriate because of the nonparallel implant, but this has disadvantages in terms of cost, manufacturing process, and prosthesis maintenance. ${ }^{9}$ The off axial occlusal load leads to denture rotation around the attachment on resorbed alveolar ridges. And ultimately causes retention loss because of matrix wear and contortion. ${ }^{18}$

The present in-vitro study evaluate retention and wear changes after 1400 cyclic loading cycle which is equivalent to one year of function. An article subjects the retentive cap to 5000 insertionremoval cycles, that resemble 4 years of withdrawal and inserting the overdenture three times a day, but the study results revealed change in the attachment retention in the first year and after one year there were little change and the statistical curves were plateau, moreover, some clinical trials studied the relationship between stud attachment retention and mastication loading, they reported that mastication deforms the attachment regardless of attachment system, the need to maintain the prosthesis during the first year is higher than in other periods. ${ }^{10}$

In the present study ,the statistics reveals that in comparison between both groups there was insignificant difference $\mathrm{P}>0.05$ at baseline and after 740cycle, while revealed significant difference after 1400 cycle $\mathrm{P}<0.05$, in other words equator with smart box group was significantly higher than ball group after 1400 cycle of cyclic loading. Trakas et al reported that this may be the reason of higher friction forces and wear patterns of the female internal components of the ball attachment that resulting in more retention loss of ball attachment. ${ }^{6}$

The present study show that Retention loss percentage for Equator attachment was $26 \%$ while for ball attachment retention \% loss was $62.5 \%$ after 1400 cycle , a previous clinical study reported that ball attachment lose $30-50 \%$ of their initial retention after 2 to 3 months, the retention loss was higher during the initial months of simulated clinical usage $^{6}$. This phenomenon could be a result of increased deformation in nylon inserts during the initial cycles. This result in accordance with our result

$\mathrm{Al} \mathrm{Gafli}$ et al reported that ball attachments on implants angulated at 5 degrees showed the least retention loss. They concluded that a small offset from the vertical axis may be beneficial in terms of attachment resistance against retention loss.5-degree angulation probably provides an ideal friction between the patrix and the matrix components, without nylon wear acceleration and presented the shortest period of time before attachment system components required replacement. As the angulation increases from 5 to 20 degrees, the wear of the ball attachments' nylon components occurs more quickly. ${ }^{11,12}$ Therefore, in our study, the degree of implant angulation was selected to be 10 degree to evaluate the amount of attachment wear. 
A systematic review 2020 concluded that if the implants are nonparallel with angulation more than 15 degree so stud attachment shouldn't be used, the main drawback of ball attachment that it loss retention gradually and must be replaced periodically, although ball attachment are resilient but placing the ball in position which is not perpendicular to the occlusal plane, influences the free movement of the attachment and make it nonresilient. ${ }^{2,22}$

The Equator smart box system introduced by Rhein 83 in 2007 . The OT equator is characterized by its low profile (vertical profile of $2.1 \mathrm{~mm}$ with a $4.4 \mathrm{~mm}$ diameter) This attachment can be used for both the overdenture with direct connection and for the overdenture to connect a secondary structure, Rhien claimed that the Smart Box Metal Housing system allows to compensate up to $50^{\circ}$ of misalignment among the dental implants. Its mechanical internal design allow a passive insertion of the denture keeping the perfect functionality of the elastic components providing comfort and functionality in implant-retained overdenture treatments ${ }^{18-20}$. The attachment mechanism mentioned interpret our study result

Tallarico et al conducted retrospective comparative study between the retention feature of the Locator and OT Equator, the study reported that the attachments retention derived from frictional contact, which is as result of a dimensional misfit between the slightly oversized male and the smaller diameter of the female abutment in addition it proved that OT Equator provide good clinical advantages, a less number of complications and less prosthetic failure can be expected ${ }^{2}$

A possible explanation of our result could be that the retentive caps of the smart box equator are made of elastic material that seems to work better than rigid one moreover the smaller size of the OT Equator may allow for an improved design of the overdenture. Furthermore, by exploiting the low profile of the OT Equator, it let the prosthodontist easily manage the limited prosthetic spaces cases which leads to a better aesthetic result ${ }^{15,16}$
A study was conducted by Yange reported that the ball attachment had deformation more than in the equator. Some authors concluded that deformation of the plastic part of the attachment is not only due to the action of placing and removing the overdenture, but may be due to the simple usage of the prosthesis in the normal daily routine. ${ }^{4,17}$

Our result represent regarding retention loss percentage loss ,revealed no statistical significance after 1400 cycle which is equal 1 year of function this could be due to the small sample size but it could reflect clinical significance as the durability and the long acting of the attachment up to 1year is important in practice and for patient satisfaction

\section{CONCLUSION}

Within the limitations of this study, the following conclusions can be drawn:. OT equator with smart box attachment has higher initial retentive power value than the ball attachment, and it should be used when implants are nonparallel.

Thus, for the greater retention especially with non-parallel implant, OT equator with smart box attachment should be preferred as the choice of attachment system for implant overdentures.

\section{REFERENCE}

1. Rutkunas V, Mizutani H, Takahashi H, Iwasaki N. Wear Simulation Effects On Overdenture Stud Attachments. Dent Mater J .2011;30: 845-53

2. Tallarico, Rodriguez Y Baena, R.., Ortensi, L.,Martinolli, M., Casucci, A.; Ferrari, E., Malaguti, G.; Montanari, M., Scrascia, R.,Vaccaro, G.,Venezia, P., Xhanari, E., Implant Overdentures Delivered With Different Design And Attachment Systems: Results Between One And 17 Years Of Follow-Up. Dent. J. 2018; 6, 71

3. Cune, M., Van Kampen, F., Van Der Bilt, A. \& Bosman, F. Patient Satisfaction And Preference With Magnet, BarClip And Ball-Socket Retained Mandibular Implant Overf dentures: A Cross-Over Clinical Trial. The International Journal of Prosthodontics. 2005; 18: 99- 105.

4. Yang Tc, Maeda Y, Gonda T, Kotecha S. Attachment Systems for Implant Overdenture: Influence of Implant 
Inclination on Retentive and Lateral Forces. Clin Oral Implants Res. 2011; 22:1315-9.

5. Al-Ghafli, S.A.; Michalakis, K.X.; Hirayama, H.; Kang, K. The In Vitro Effect of Different Implant Angulations and Cyclic Dislodgement on the Retentive Properties of an Overdenture Attachment System. J. Prosthet. Dent. 2009; $102,140-147$

6. Trakas T, Michalakis K, Kang K, Hirayama H. Attachment Systems for Implant Retained Overdentures: A Literature Review. Implant Dent 2006; 15:24-34

7. Abi Nader S, De Souza Rf, Fortin D, De Koninck L, Fromentin O, Albu- Querque Junior Rf. Effect Of Simulated Masticatory Loading On The Retention Of Stud Attachments For Implant Overdentures. J Oral Rehabil 2011; 38:157-64.

8. Ángel Vicente-Escuder Eduardo J. Selva-Otaolaurruchi in Vitro Retention Capacity of Two Overdenture Extra Systems: Locator ${ }^{\circledR}$ And Equatorj Clin Exp Dent. 2018; 10: 681-6.

9. Alsabeeha Nh , Payne Ag, Swain Mv. Attachment Systems For Mandibular Two-Implant Overdentures: A Review Of In Vitro Investigations On Retention And Wear Features. Int J Prosthodont 2009; 22:429-40.

10. Nayrouz A. Metwally, Ahmed M. Abdel-Hamid, Ahmed M, Nermeen A. Conservative Approach For Management Of Posterior Atrophic Maxilla Using Implant Assisted Overdenture Clincal Trial. Alexandria Dental Journal. $2020 ; 3,81-87$

11. Awad Ma, Lund Jp, Dufresne E, Et Al. Comparing the Efficacy of Mandibular Implant-Retained Overdentures and Conventional Dentures among Middle-Aged Edentulous Patients: Satisfaction and Functional Assessment. Int J Prosthodont: 2003; 16; 117-122.

12. Doundoulakis Jh, Eckert Se, Lindquist Cc, Jeffcoat Mk. The Implant-supported Overdenture as an Alternative to the complete mandibular denture. J Am Dent Assoc. 2003 ; 11: $1455-8$

13. Elsyad Ma, Eltowery Sm, Gebreel Aa. Peri-Implant Strain around Mesially Inclined Two-Implant-Retained Mandib- ular Overdentures with Locator Attachments. J Oral Sci. 2017; 59(4):483-490.

14. Yang T-C, Maeda Y, Gonda T, Kotecha S. Attachment Systems for Implant Overdenture: Influence of Implant Inclination on Retentive and Lateral Forces.Clin. Oral Impl. Res. 22, 2011; 1315-1319.

15. Ahmad R, Abu-Hassan Mi, Chen J, Et Al. The Relationship of Mandibular Morphology With Residual Ridge Resorption Associated With Implant-Retained Overdentures. Int J Prosthodont. 2016; 29(6):573-580

16. Scherer Md. Presurgical Implant-Site Assessment and Restoratively Driven Digital Planning. Dent Clin North Am 2014; 58(3):561-595.

17. Frits K, Marco C, Andries V,Frits B. Retention And Postinsertion Maintenance of Bar-Clip, Ball And Magnet Attachments In Mandibular Implant Overdenture Treatment: An In Vivo Comparison After 3 Months of Function Clin. Oral Impl. Res. 14, 2003; 720-726

18. Yoo Js, Kwon Kr, Noh K, Lee H, Paek J. Stress Analysis of Mandibular Implant Overdenture With Locator And Bar/Clip Attachment: Comparative Study With Differences In The Denture Base Length. J Adv Prosthodont.. 2017; 9(3):143-151.

19. Daou Ee. Biomaterial Aspects: A Key Factor In The Longevity Of Implant Overdenture Attachment Systems. J Int Soc Prev Community Dent.; 2015; 5(4):255-262.

20. Kurtulus IL, Gurbulak AG. The In Vitro comparison of the retention of an implant-supported stud attachment locator and straumann ball attachment at different angulations. Niger J Clin Pract .2018;21: 639-44.

21. Mínguez-Tomás, N., Alonso-Pérez-Barquero, J., FernándezEstevan, L., Vicente-Escuder, Á., \& Selva-Otaolaurruchi, E. J.. In vitro retention capacity of two overdenture attachment systems: Locator® and Equator®. Journal of Clinical and Experimental Dentistry. 2018;10(7), 681-e686

22. Ikbal, M., Mude, A. H., Dammar, I., Launardo, V., \& Sudarman, I. A. Locator or ball attachment systems for mandibular implant overdentures: A systematic review. Systematic Reviews in Pharmacy, (2020) 11(9), 15-19. 